\title{
Comparative Study of Clinical and Polysomnographic Characteristics of Obstructive Sleep Apnea Among Obese and Non-Obese Patients at Chiang Mai University Hospital
}

\author{
Theerakorn Theerakittikul ${ }^{* 1,2}$, Nattinee Laksananun ${ }^{3}$, Juthamas Inchai ${ }^{1}$, Chaicharn Pothirat ${ }^{1}$, Chalerm \\ Liwsrisakun ${ }^{1}$, Chaiwat Bumroongkit ${ }^{1}$, Athavudh Deesomchok ${ }^{1}$, Atikun Limsukon ${ }^{1}$ and Pattraporn \\ Tajaroenmuang ${ }^{1}$ \\ ${ }^{1}$ Department of Internal Medicine, Faculty of Medicine, Division of Pulmonary-Critical Care and Allergy, Chiang Mai University, Chiang \\ Mai, Thailand \\ ${ }^{2}$ Sleep Disorder Center, Center for Medical Excellence, Chiang Mai University, Chiang Mai, Thailand \\ ${ }^{3}$ Department of Internal Medicine, Faculty of Medicine, Chiang Mai University, Chiang Mai, Thailand
}

*Corresponding author: Theerakorn Theerakittikul, Department of Internal Medicine, Faculty of Medicine, Division of Pulmonary-

Critical Care and Allergy, Chiang Mai University, Chiang Mai, Thailand

\section{ARTICLE INFO}

Received: 蔧 January 18, 2019

Published: 幽 February 19, 2019

Citation: Theerakorn T, Nattinee L, Juthamas I, Chaicharn P, Chalerm L, et al. Comparative Study of Clinical and Polysomnographic Characteristics of Obstructive Sleep Apnea Among Obese and Non-Obese Patients at Chiang Mai University Hospital. Biomed J Sci \& Tech Res 14(5)-2019. BJSTR. MS.ID.002608.

Abbreviations: OSA: Obstructive Sleep Apnea; UAO: Upper Airway Obstruction; CAD: Coronary Artery Disease; EDS: Excessive Day Time Sleepiness; ESS: Epworth Sleepiness Scales; AHI: Apnea-Hypopnea Index; COPD: Chronic Obstructive Pulmonary Disease; BMI: Body Mass Index; FTP: Friedman Tongue Position; PLMI: Periodic Leg Movement Index; SD: Standard Deviation

\section{ABSTRACT}

Background: Recent studies have shown that obstructive sleep apnea (OSA) is common in both obese and nonobese patients with different clinical and polysomnographic features in each group. However, such studies were scarce and diverse, with small sample sizes, and were rarely conducted in Asian populations. Only one trial to date has employed the classification of BMI in adult Asians by WHO criteria which is currently used as a reference based on morbidity risk.

Objectives: To compare the clinical, anthropometric and polysomnographic characteristics of non-obese and obese patients with OSA.

Methods: Data from OSA patients at Chiang Mai University Hospital Sleep Clinic from January 2013 to December 2015 were reviewed, including clinical characteristics, anthropometric measurements, and polysomnographic parameters. Patients were stratified into obese and non-obese groups, using BMI $\geq 25 \mathrm{~kg} / \mathrm{m}^{2}$ as the cutoff for obesity.

Results: Of the total 418 patients analyzed, 295 (70.6\%) were obese. Mean age was higher in the non-obese group ( $59.14 \pm 13.48 \mathrm{yr}$ vs $55.16 \pm 13.33 \mathrm{yr}, \mathrm{p} 0.020)$. Diabetes mellitus and hypertension were more common in the obese group, and obese patients had larger neck circumference $(15.71 \pm 1.54$ in vs $13.94 \pm 1.10 \mathrm{in}, \mathrm{p}<0.001)$ and waist circumference ( $41.26 \pm 5.37$ in vs $34.66 \pm 3.43$ in, $p<0.001)$. There were no differences in any other comorbidities, ESS, EDS or other clinical parameters. Regarding anthropometric measurements, obese patients had higher Friedmann tongue position scores ( $\mathrm{p}<0.001)$, while micrognathia and retrognathia were more prevalent in non-obese patients $(27.3 \%$ vs $7.6 \%, \mathrm{p}<0.001$, and $3.3 \%$ vs $0.3 \%$, p 0.028 , respectively). Regarding polysomnographic recordings, obese patients had significantly worse parameters demonstrated by higher NREM AHI ( $87.31 \pm 31.08$ vs $67.71 \pm 27.70)$, desaturation index (5.93 \pm 6.07 vs $2.50 \pm$ 3.55 ), more total sleep time with oxygen saturation $<90 \%$ (10.83\% vs $3.08 \%)$, more CPAP use $(94.8 \%$ vs $85.1 \%)$ with higher CPAP pressure (11.17 \pm 4.02 vs $8.65 \pm 4.62 \mathrm{cmH} 20)$, but lower minimal oxygen saturation $(83.00 \%$ vs $88.08 \%)$. Multivariate logistic regression analysis showed that only age, neck and waist circumferences, micrognathia, desaturation index, and CPAP use were significantly and independently associated with OSA in the nonobese population.

Conclusion: OSA is not restricted to obese populations. Non-obese OSA patients in this study were older, had smaller neck and waist circumferences, and were more like to have micrognathia. Lower OSA severity was demonstrated in non-obese patients by most polysomnographic parameters, especially the desaturation index, and less CPAP use. 


\section{Background}

Obstructive sleep apnea (OSA) is a disease characterized by repetitive sleep disruptions due to episodes of upper airway obstruction (UAO), resulting in nocturnal hypoxemia, sleep termination with frequent arousals, and excessive daytime sleepiness [13]. OSA is more common than generally believed. It can also be a serious and life-threatening disorder, which may lead to adverse cardiovascular consequences and increased mortality. Obesity has been recognized as one of the classical risk factors of OSA, with up to $60 \%$ of OSA patient being obese, and up to $40 \%$ of the obese population carrying a diagnosis of OSA [4]. This may be due to anatomical alterations in obesity predisposing to UAO or collapse during sleep. Recent studies have shown that OSA is not uncommon in the non-obese population, and many have proposed some different characteristics of OSA in this population in regard to clinical, cephalometric and polysomnographic features, suggesting two different disease entities among the OSA population. For non-obese OSA patients, medical comorbidities (e.g. diabetes mellitus, hypertension, and coronary artery disease (CAD), smoking, alcohol use and sedative drug use) were less prevalent. As for cepholometric parameters, thyromental distance was significantly shorter in this population. However, differences in other factors, including adenotonsillar hypertrophy, neck circumference, and symptoms of excessive day time sleepiness (EDS) evaluated by the Epworth Sleepiness Scales (ESS) did not reach statistical significance. On the other hand, OSA was more severe in obese patients as demonstrated by most polysomnographic parameters including the apnea-hypopnea index (AHI) [4-10]. However, related studies were scarce and diverse, with small samples, were rarely conducted in Asian populations. Only one study to date [5] has employed the proposed classification of weight by BMI in adult Asians by WHO (WPRO, 2000), using BMI $\geq 25 \mathrm{~kg} / \mathrm{m}^{2}$ as the cutoff point for obesity, which is currently used as a reference based on the risk of morbidities $[11,12]$.

\section{Objectives}

This study was designed to evaluate the differences in clinical, anthropometric and polysomnographic characteristics between non-obese and obese patients with OSA in a Thai population.

\section{Material and Methods}

This retrospective observational study was done by reviewing data from all patients aged $\geq 16$ years referred to Chiang Mai University Hospital Sleep Clinic for suspected OSA who underwent polysomnography confirmation from January 2013 to December 2015. OSA was diagnosed by polysomnography when AHI was > 5 events/h with consistent clinical symptoms, including frequent snoring or EDS. This study was conducted under approval by the local research ethics committee. Patients with pregnancy, uncontrolled congestive heart failure or chronic obstructive pulmonary disease (COPD) were excluded from the study. Data regarding clinical characteristics, anthropometric measurements and polysomnographic parameters was collected from the medical record with review of the polysomnographic report by the authors, including: a) Baseline clinical characteristics: age, sex, associated comorbidities (DM, HT, DLP, CAD, DCM, CVA, asthma, and COPD) and clinical presentation of OSA (EDS, Epworth Sleepiness Scale (ESS), snoring, morning headache, apnea, awakening, and nocturia).

b) Anthropometric measurements: height, body weight, body mass index (BMI), neck and waist circumferences, Friedman Tongue Position (FTP) classified into grade I-IV, presence of adenotonsillar hypertrophy and grading from $0-4$, long uvula, high arch palate, micrognathia, and retrognathia.

c) Polysomnographic parameters: total, REM, and NREM $\mathrm{AHI}$, respiratory arousal index, desaturation index, minimal oxygen saturation, total sleep time with oxygen saturation $<90 \%$, periodic leg movement index (PLMI), CPAP use, and CPAP pressure.

Patients were stratified into obese and non-obese groups, using BMI $\geq 25 \mathrm{~kg} / \mathrm{m}^{2}$ as the cutoff for obesity. The primary aim of the study was to compare the clinical characteristics, cephalometric measurements and polysomnographic findings between non-obese and obese patients with OSA. The secondary aim was to determine the percentage of OSA patients that were non-obese.

\section{Statistical Analysis}

All statistical analysis of the data was performed using SPSS version 22.0(SPSS Inc., Chicago, USA). The quantitative variables were expressed as mean \pm standard deviation (SD), and the qualitative variables as frequencies and percentages. $\chi^{2}$ test was used to evaluated categorical data. Numerical data which exhibited normal distribution was analyzed using the student t-test, while data that was not normally distributed was analyzed by the non-parametric Mann-Whitney U test. The Fisher Exact test was used for data with an expected frequency of variable $\leq 5$. In order to determine the relationship between the study variables and OSA, multivariate logistic regression analysis was performed. A P value of $<0.05$ was considered statistically significant in all analyses.

\section{Results}

During the study period, 421 patients were evaluated at Chiang Mai University Sleep clinic for suspected OSA and had polysomnography performed. Three of these patients were excluded due to missing data (on BMI). A total of 418 patients remained for analysis, of these, 295 (70.6\%) were obese and most patients were male $(62.2 \%)$. Mean age was significantly higher in the non-obese group $(59.14 \pm 13.48$ yr vs $55.16 \pm 13.33$ yr, p 0.020). Diabetes mellitus and hypertension were more prevalent in the obese group $(20.4 \%$ vs $9.1 \%$, p 0.006 , and $48.4 \%$ vs $30.6 \%$, p 0.001 , respectively). However, there were no differences in any other comorbidities, ESS, EDS or other clinical parameters Table 1. Regarding anthropometric measurements, larger neck circumference $(15.71 \pm 1.54$ in vs 13.94 \pm 1.10 in, $p<0.001$ ) and waist circumferences $(41.26 \pm 5.37$ in vs $34.66 \pm 3.43$ in, $p<0.001$ ) were noted in the obese patients. Also, in the obese population, the Friedmann Tongue Position (FTP) score 
was significantly higher $(\mathrm{p}<0.001)$, while micrognathia and retrog- other parameters including adenotonsilar hypertrophy, tonsil size, nathia were more common in the nonobese group $(27.3 \%$ vs $7.6 \%$, and presence of a long uvula or high arched palate did not reach $\mathrm{p}<0.001$, and $3.3 \%$ vs $0.3 \%$, p 0.028, respectively). Differences in statistical significance Table 2.

Table 1: Baseline clinical characteristics, comorbidities and clinical presentation in non-obese and obese OSA patients.

\begin{tabular}{|c|c|c|c|}
\hline & Non-Obese OSA $(\mathrm{N}=123)$ & Obese OSA ( $\mathrm{N}=295)$ & $P$ Value \\
\hline \multicolumn{4}{|l|}{ Clinical characteristics } \\
\hline Males & $78(63.4 \%)$ & $182(61.2 \%)$ & 0.825 \\
\hline Age (years) & $59.14 \pm 13.48$ & $55.16 \pm 13.33$ & 0.020 \\
\hline \multicolumn{4}{|l|}{ Comorbidities } \\
\hline DM & $11(9.1 \%)$ & $59(20.4 \%)$ & 0.006 \\
\hline HT & $37(30.6 \%)$ & $140(48.4 \%)$ & 0.001 \\
\hline DLP & $27(22.3 \%)$ & $80(27.7 \%)$ & 0.270 \\
\hline CAD & $6(5.0 \%)$ & $17(5.9 \%)$ & 0.817 \\
\hline DCM & $0(0.0 \%)$ & $2(0.7 \%)$ & 0.583 \\
\hline CVA & $2(1.7 \%)$ & $16(5.5 \%)$ & 0.111 \\
\hline Asthma & $14(11.6 \%)$ & $52(18.0 \%)$ & 0.140 \\
\hline COPD & $3(2.5 \%)$ & $9(3.1 \%)$ & 0.768 \\
\hline \multicolumn{4}{|l|}{ Clinical presentation of OSA } \\
\hline Epworth sleepiness scales (ESS) & $8.60 \pm 5.16$ & $9.64 \pm 5.41$ & 0.239 \\
\hline Excessive daytime sleepiness (EDS) & $100(83.3 \%)$ & $240(83.3 \%)$ & 1.000 \\
\hline Snoring & $112(92.6 \%)$ & $276(95.5 \%)$ & 0.236 \\
\hline Morning headache & $34(28.1 \%)$ & $95(32.9 \%)$ & 0.354 \\
\hline Apnea & $30(24.8 \%)$ & 90 (31.1\%) & 0.234 \\
\hline Awakening & 65 (53.7\%) & $164(56.7 \%)$ & 0.587 \\
\hline Nocturia & $46(38.0 \%)$ & 127 (43.9\%) & 0.275 \\
\hline
\end{tabular}

Table 2: Anthropometric characteristics in non-obese and obese OSA patients.

\begin{tabular}{|c|c|c|c|}
\hline & Non-Obese OSA $(\mathrm{N}=123)$ & Obese OSA(N=295) & $P$ Value \\
\hline Neck circumference (inches) & $13.94 \pm 1.10$ & $15.71 \pm 1.54$ & $<0.001$ \\
\hline Waist circumference (inches) & $34.66 \pm 3.43$ & $41.26 \pm 5.37$ & $<0.001$ \\
\hline \multicolumn{4}{|l|}{ Friedman Tongue Position (FTP) } \\
\hline FTP I & $15(12.4 \%)$ & $31(10.7 \%)$ & $<0.001$ \\
\hline FTP II a & $13(10.7 \%)$ & $12(4.2 \%)$ & \\
\hline FTP II b & $35(28.9 \%)$ & $38(13.1 \%)$ & \\
\hline FTP III & $54(44.6 \%)$ & $145(50.2 \%)$ & \\
\hline FTP IV & $4(3.3 \%)$ & $63(21.8 \%)$ & \\
\hline Aden tonsillar hypertrophy & $3(2.5 \%)$ & $14(4.9 \%)$ & 0.299 \\
\hline \multicolumn{4}{|l|}{ Tonsil size } \\
\hline Grade 0 & $118(97.5 \%)$ & $274(94.8 \%)$ & 0.282 \\
\hline Grade 1 & $3(2.5 \%)$ & $6(2.1 \%)$ & \\
\hline Grade 2 & $0(0.0 \%)$ & $8(2.8 \%)$ & \\
\hline Grade 3 & $0(0.0 \%)$ & $1(0.3 \%)$ & \\
\hline Grade 4 & $0(0.0 \%)$ & $0(0.0 \%)$ & \\
\hline Micrognathia & $33(27.3 \%)$ & $22(7.6 \%)$ & $<0.001$ \\
\hline Retrognathia & $4(3.3 \%)$ & $1(0.3 \%)$ & 0.028 \\
\hline Long uvula & $1(0.8 \%)$ & $2(0.7 \%)$ & 1 \\
\hline High arch palate & $3(2.5 \%)$ & $1(0.3 \%)$ & 0.079 \\
\hline
\end{tabular}


Regarding polysomnographic findings, most patients in both groups had severe OSA, represented by mean total AHI of $88.26 \pm$ 29.72 and $69.31 \pm 25.17$ in obese and non-obese patients, respectively, and the majority (92\%) were using CPAP. Obese OSA patients had significantly worse polysomnographic parameters as demonstrated by higher NREM AHI (87.31 \pm 31.08 vs $67.71 \pm 27.70)$, desaturation index ( $5.93 \pm 6.07$ vs $2.50 \pm 3.55)$, more total sleep time with oxygen saturation $<90 \%(10.83 \%$ vs $3.08 \%)$, more CPAP use (94.8\% vs $85.1 \%)$ with higher CPAP pressure $(11.17 \pm 4.02$ vs 8.65

Table 3: Polysomnographic characteristics in non-obese and obese OSA patients.

\begin{tabular}{|c|c|c|c|}
\hline & Non-Obese OSA $(\mathrm{N}=123)$ & Obese OSA $(\mathrm{N}=295)$ & $P$ Value \\
\hline Total AHI (events/h) & $69.31 \pm 25.17$ & $88.26 \pm 29.72$ & 0.064 \\
\hline REM AHI (events/h) & $5.54 \pm 16.32$ & $7.91 \pm 23.22$ & 0.064 \\
\hline NREM AHI (events/h) & $67.71 \pm 27.70$ & $87.31 \pm 31.08$ & $<0.001$ \\
\hline Respiratory arousal index & $42.41 \pm 19.27$ & $42.96 \pm 20.80$ & 0.776 \\
\hline Desaturation index & $2.50 \pm 3.55$ & $5.93 \pm 6.07$ & $<0.001$ \\
\hline Minimal oxygen saturation (\%) & $88.08 \pm 5.71$ & $83.00 \pm 10.12$ & $<0.001$ \\
\hline Total sleep time with oxygen saturation<90\% (\%) & $3.08 \pm 10.06$ & $10.83 \pm 20.62$ & $<0.001$ \\
\hline Periodic leg movement index & $4.50 \pm 10.54$ & $3.30 \pm 10.16$ & 0.64 \\
\hline CPAP use & $103(85.1 \%)$ & $276(94.8 \%)$ & 0.002 \\
\hline CPAP pressure & $8.65 \pm 4.62$ & $11.17 \pm 4.02$ & $<0.001$ \\
\hline
\end{tabular}

Table 4: Results of the multivariate analysis of potential variables for OSA in non-obese patients compared to obese patients.

\begin{tabular}{|c|c|c|c|}
\hline Variables & Odd Ratio (OR) & $95 \% \mathrm{CI}$ & $P$ Value \\
\hline Age & 1.062 & $1.030-1.095$ & $<0.001$ \\
\hline Neck circumference & 0.644 & 0.463-0.895 & 0.009 \\
\hline Waist circumference & 0.743 & $0.659-0.838$ & $<0.001$ \\
\hline Micrognathia, presence of & 4.181 & $1.473-11.864$ & 0.007 \\
\hline Desaturation index & 0.885 & $0.806-0.972$ & 0.011 \\
\hline CPAP use & 0.285 & $0.093-0.870$ & 0.028 \\
\hline
\end{tabular}

\section{Discussion}

Many previous studies have shown that OSA is not restricted to the obese population, with up to $40 \%$ of patients being non-obese [4, 6-8]. A recent study in Thailand [5], on the contrary, discovered that the majority of OSA patients were non-obese (63.4\%). Our study finding that $39.4 \%$ of OSA patients under evaluation were non-obese is in concordance with the previous literature. Regarding comorbidities, hypertension, dyslipidemia and coronary artery disease have been shown to be more prevalent in obese compared to non-obese patients in several studies $[5,8]$. Hypertension, obesity and OSA can either co-exist or contribute to the progression of one other, leading to resistant hypertension and adverse cardiovascular outcomes. Our finding of higher hypertension prevalence in the obese patients was similar; in addition, diabetes mellitus was also more common in this group, which the relationship between obesity and insulin resistance and metabolic syndrome may be responsible for. Obese patients with OSA are known to have greater $\pm 4.62 \mathrm{cmH} 20)$, but lower minimal oxygen saturation $(83.00 \%$ vs $88.08 \%$ ) Table 3. Multivariate logistic regression analysis showed that only increasing age (OR 1.062, p <0.001), lower neck and waist circumferences (OR 0.644, p 0.009, and OR 0.743, p < 0.001, respectively), presence of micrognathia (OR 4.181, p 0.007), lower desaturation index (OR 0.885, p 0.011), and more CPAP use (OR 0.285, p 0.028 ) were significantly and independently associated with OSA in the non-obese population Table 4. 
characteristics, obesity is a principle risk factor for OSA due to the anatomical alterations that predispose obese individuals to upper airway obstruction during sleep. Increased soft tissue surrounding the pharyngeal airway within a limited bony closure leads to airway narrowing and collapse. Increased tongue volume from accumulated fat and anterior displacement of the hyoid bone, related to obesity, as well as larger neck circumferences with increased parapharyngeal fat also increase the tendency for upper airway obstruction $[9,10]$. Larger neck circumference in obese OSA patients compared to non-obese patients in our study supports these previous findings. Furthermore, larger waist circumference in this population as seen in this study is a marker of obesity, especially truncal obesity with increased visceral fat.

On the other hand, non-obese patients with OSA represent a different OSA entity. A study from Sakakibara et al. [9] found the bony structure discrepancies to be a major contributing factor. Short anterior cranial base and mandibular length are the two main predictors [9], accompanied by caudal hyoid, increased soft palate soft tissue, decreased pharyngeal width with airway narrowing at the soft palate level, oropharyngeal and nasopharyngeal narrowing [10]. Higher prevalence of micrognathia and retrognathia in the non-obese OSA group in our study supports findings from previous literature, since such alterations can similarly cause cephalometric imbalance by shortening the length of oropharynx and crowding the upper airway structure.

There were some potential limitations to this study. First, the selection of patients may have been biased. Since our study population was a selected group of patients referred to the sleep clinic for evaluation of suspected OSA, the findings in this study may not be generalizable to the population of patients with milder OSA severity, or those with lower probability of OSA presenting before confirmatory polysomnographic evaluation. Second, this is a retrospective study, with all the implications that this entails. Potential strengths of this study include the relatively large sample size and the large number of variables collected for characteristic studied.

\section{Conclusion}

OSA is not uncommon in the non-obese population. Non-obese patients tend to be older, with smaller neck and waist circumferences, and micrognathia as a more prevalent feature. Lower OSA severity was demonstrated by most polysomnographic parameters, especially the desaturation index, and less CPAP use in non-obese patients.

\section{Acknowledgement}

The authors would like to acknowledge Dr. Megan Brandeland for her review and comments.

\section{References}

1. McNicholas WT (1996) Diagnostic criteria for the sleep apnoea syndrome: time for consensus. The European respiratory journal. Apr 9(4): 634-635.

2. Medicine AAoS (2005) International classification of sleep disorders 2nd ed: Diagnostic and coding manual. Westchester IL: American Academy of Sleep Medicine.

3. Park JG, Ramar K, Olson EJ (2011) Updates on definition, consequences, and management of obstructive sleep apnea. Mayo Clinic proceedings. Jun 86(6): 549-554.

4. Chierakul N, Chaipattarapol C, Ruttanaumpawan P, Nana A, Naruman C, et al. (2007) Comparison of clinical and polysomnographic characteristics of non-obese and obese patients with obstructive sleep apnea. Journal of the Medical Association of Thailand Chotmaihet thangphaet. Nov 90, 2: 48-53.

5. Chirakalwasan N, Teerapraipruk B, Simon R, Hirunwiwatkul P, Jaimchariyatam N, et al. (2013) Comparison of polysomnographic and clinical presentations and predictors for cardiovascular related diseases between non-obese and obese obstructive sleep apnea among Asians. Journal of clinical sleep medicine JCSM official publication of the American Academy of Sleep Medicine. Jun 15 9(6): 553-537.

6. Dacal Quintas R, Tumbeiro Novoa M, Alves Perez MT, Santalla Martinez ML, Acuna Fernandez A, et al. (2013) Obstructive sleep apnea in normal weight patients: characteristics and comparison with overweight and obese patients. Archivos de bronconeumologia. Dec 49(12): 513-517.

7. Garg R, Singh A, Prasad R, Saheer S, Jabeed P, et al. (2012) A comparative study on the clinical and polysomnographic pattern of obstructive sleep apnea among obese and non-obese subjects. Annals of thoracic medicine. Jan 7(1): 26-30.

8. Rey de Castro J, Rosales Mayor E (2011) [Clinical and polysomnographyc differences between obese and non-obese patients with obstructive sleep apnea-hypopnea syndrome]. Revista peruana de medicina experimental y salud publica. Diferencias clinicas y polisomnograficas entre obesos y no obesos con sindrome de apneas-hipopneas del sueno. Dec 28(4): 595-601.

9. Sakakibara H, Tong M, Matsushita K, Hirata M, Konishi Y, et al. (1999) Cephalometric abnormalities in non-obese and obese patients with obstructive sleep apnoea. The European respiratory journal 13(2): 403410 .

10. Yu X, Fujimoto K, Urushibata K, Matsuzawa Y, Kubo K, et al. (2003) Cephalometric analysis in obese and nonobese patients with obstructive sleep apnea syndrome. Chest 124(1): 212-218.

11. (2004) Consultation WHOE Appropriate body-mass index for Asian populations and its implications for policy and intervention strategies. Lancet. 363(9403): 157-163.

12. (2000) World Health Organization ROftWPW, International Association for the Study of Obesity, International Obesity Task Force. The AsiaPacific Perspective: Redefining obesity and its treatment. Health Communications Australia Pty Ltd. 
ISSN: 2574-1241

DOI: 10.26717.BJSTR.2019.14.002608

Theerakorn Theerakittikul. Biomed J Sci \& Tech Res

(C) (i) This work is licensed under Creative

Submission Link: https://biomedres.us/submit-manuscript.php

$\begin{array}{ll}\text { BIOMEDICAL } & \text { Assets of Publishing with us } \\ \text { RESEARCHES } & \text { - Global archiving of articles } \\ & \text { - Immediate, unrestricted online access } \\ & \text { - Rigorous Peer Review Process } \\ \end{array}$

